\title{
ASSISTÊNCIA DE AR E VOLUMES DE APLICAÇÃO NA DEPOSIÇÃO DE CALDA E NO CONTROLE DO ARROZ VERMELHO (Oryza sativa L.) ${ }^{1}$
}

\author{
LEOPOLDO L. S. VIGANO ${ }^{2}$, CARLOS G. RAETANO ${ }^{3}$
}

RESUMO: O trabalho teve como objetivo avaliar o efeito da assistência de ar junto à barra pulverizadora e de três volumes de pulverização na dessecação e deposição da calda em arroz vermelho, sob cultivo de nabo forrageiro, em áreas de recuperação de várzeas, utilizando o herbicida paraquat e o corante Azul Brilhante, respectivamente. Os volumes de pulverização foram 100; 200 e $300 \mathrm{~L} \mathrm{ha}^{-1}$ da solução aquosa, contendo corante alimentício $\left(1.500 \mathrm{mg} \mathrm{L}^{-1}\right)$. Com ou sem a assistência de ar junto à barra, foram utilizadas pontas de pulverização de jato plano tipo AXI 110015 à pressão de 117,3 kPa, AXI 11002 e AXI 11003 a 276 kPa. A avaliação da deposição da pulverização deu-se em folhas de plantas de arroz vermelho. Os maiores volumes (200 e $300 \mathrm{~L} \mathrm{ha}^{-1}$ ) pulverizados com a assistência de ar junto à barra pulverizadora proporcionaram maiores depósitos do corante em relação ao volume de $100 \mathrm{~L} \mathrm{ha}^{-1}$. Não foram constatadas diferenças na deposição do corante para os volumes pulverizados, sem a assistência de ar junto à barra, tampouco entre os volumes de 200 e $300 \mathrm{~L} \mathrm{ha}^{-1}$ com a assistência de ar junto à barra. As maiores percentagens de controle do arroz vermelho foram obtidas com a assistência de ar junto à barra, independentemente do volume pulverizado, equivalendo-se ao controle obtido com $300 \mathrm{~L} \mathrm{ha}^{-1}$, sem o uso dessa tecnologia.

PALAVRAS-CHAVE: tecnologia de aplicação, controle químico, herbicida.

\section{AIR ASSISTANCE AND VOLUME OF APPLICATION IN SPRAY DEPOSITION AND IN RED RICE CONTROL (Oryza sativa L.)}

\begin{abstract}
The aim of this research was to evaluate the effect of air-assistance on spraying at three volumes in spray deposition and control of red rice under fodder radish cultivation. To evaluate the control of this weed and spray deposition were used paraquat herbicide and a Brilliant Blue dye, respectively. The three spraying volumes were 100, 200 and $300 \mathrm{~L} \mathrm{ha}^{-1}$, using a tracer dye at 1,500 $\mathrm{mg} \mathrm{L}^{-1}$. Both solutions and volumes were sprayed with flat fan nozzles AXI 110015 at $117.3 \mathrm{kPa}$, AXI 11002 and AXI 11003 at $276 \mathrm{kPa}$, respectively, with and without air-assistance on the boom. The evaluation of deposition was made on red rice leaves. The highest spray volumes (200 and $300 \mathrm{~L} \mathrm{ha}^{-1}$ ) permitted to obtain greater dye deposits with air assistance in the spray boom. No difference it was observed on spray deposition at 100; 200 and $300 \mathrm{~L} \mathrm{ha}^{-1}$ volumes without air assistance and at 200 as well as $300 \mathrm{~L} \mathrm{ha}^{-1}$ with air assistance. The highest percentages of red rice control were obtained with $300 \mathrm{~L} \mathrm{ha}^{-1}$ without air assistance as well as for all spray volumes using this technology.
\end{abstract}

KEYWORDS: application technology, chemical control, herbicide.

\section{INTRODUÇÃO}

A aplicação de produtos fitossanitários com assistência de ar na barra pulverizadora como forma de auxiliar a melhoria da eficiência, é comumente empregada em pomares, mas pouco utilizada em lavouras anuais (FURNESS,1991). No entanto, HISLOP (1991) menciona que, embora a idéia de usar a assistência de ar em pulverizações não seja nova, estudos mais detalhados devem ser efetuados em relação ao uso dessa tecnologia.

\footnotetext{
${ }^{1}$ Extraído da Dissertação de Mestrado do primeiro autor.

${ }^{2}$ Engo Agrônomo, Mestrando do PPGA - Agricultura, FCA/UNESP, Câmpus de Botucatu - SP, Fone: (0XX14) 3811.7167, vigano@fca.unesp.br

${ }^{3}$ Eng ${ }^{0}$ Agrônomo, Prof. Assistente Dr., Departamento de Produção Vegetal, FCA/UNESP, Botucatu - SP.

Recebido pelo Conselho Editorial em: 10-4-2007
}

Aprovado pelo Conselho Editorial em: 11-10-2007 
A assistência de ar junto à barra de pulverização pode auxiliar na redução da deriva (COOKE et al.,1990 e BAUER \& RAETANO, 2000), bem como no aumento da penetração das gotas no dossel da cultura (MATTHEWS, 2000). Por outro lado, os níveis dos depósitos em plantas-alvo não dependem somente do equipamento aplicador, mas também da idade e do tipo da cultura, da condição operacional e do tipo de ponta de pulverização (COOKE et al., 1990).

A utilização de pulverizadores dotados de assistência de ar é importante na redução dos custos com aplicação de produtos fitossanitários, uma vez que eles podem reduzir o volume de aplicação, aumentando a capacidade operacional do equipamento (STEVENSON \& JAMES, 1997).

O uso de herbicidas, no controle ou na dessecação das espécies infestantes em áreas agrícolas, tem causado a contaminação de áreas vizinhas e culturas sensíveis à aplicação (CONSTANTIN et al., 2007 e OLIVEIRA JÚNIOR et al., 2007), bem como do aplicador. Assim, a assistência de ar pode contribuir para a redução da deriva e da dose do herbicida na dessecação da comunidade de plantas infestantes (GARCIA et al., 2004). No entanto, o uso dessa tecnologia mostra-se incipiente no controle de plantas daninhas, especialmente em áreas de várzea.

A recuperação de áreas de cultivo de arroz irrigado pode ser obtida com o plantio do nabo forrageiro (Raphanus sativus L.). Porém, ao final de seu desenvolvimento vegetativo, a presença de luz favorece o aparecimento de outras plantas infestantes, a exemplo do arroz vermelho (Oryza sativa L.).

Assim sendo, o presente trabalho teve o objetivo de avaliar o efeito da assistência de ar junto à barra de pulverização combinada a três volumes de calda, na deposição da pulverização de uma substância marcadora, bem como na dessecação do arroz vermelho sob cultivo de nabo forrageiro com o herbicida paraquat.

\section{MATERIAL E MÉTODOS}

Dois experimentos foram conduzidos em área de recuperação de cultivo do arroz irrigado (várzea), localizada na Fazenda Experimental Edgárdia, da Faculdade de Ciências Agronômicas, Câmpus de Botucatu, UNESP. A localização geográfica está definida pelas coordenadas $22^{\circ} 51^{\prime} 5^{\prime}$, sul, $48^{\circ} 26^{\prime} 5^{\prime}$ ' oeste e altitude aproximada de $770 \mathrm{~m}$.

Os experimentos foram instalados em área de várzea com nabo forrageiro como cultura de rotação do arroz irrigado, após o estádio de máximo crescimento vegetativo ( 90 dias após a semeadura com $12 \mathrm{~kg}$ de sementes $\mathrm{ha}^{-1}$ ). Nessa ocasião, as plantas de $R$. sativus com altura média de $0,80 \mathrm{~m}$ e menor enfolhamento possibilitaram maior incidência de luz na superfície do solo e, conseqüentemente, a germinação natural do arroz vermelho. Essas plantas infestantes apresentavam três a quatro folhas, com altura média de $0,20 \mathrm{~m}$.

O clima de Botucatu, baseado no sistema de classificação internacional de Köeppen, foi incluído no tipo $\mathrm{Cfa}$, que significa clima temperado com inverno frio e seco, e verão quente, com temperatura do mês mais frio inferior a $18{ }^{\circ} \mathrm{C}$ e a do mês mais quente superior a $22{ }^{\circ} \mathrm{C}$.

\section{Experimento 1}

O efeito da assistência de ar combinado a volumes de pulverização sobre os depósitos da calda foi avaliado em plantas de arroz vermelho. O delineamento experimental foi o de blocos ao acaso, com seis tratamentos distribuídos no esquema fatorial $3 \times 2$, sendo três volumes de calda combinados a duas tecnologias de aplicação (Tabela 1), em quatro repetições.

O equipamento utilizado na execução do trabalho foi um pulverizador modelo Falcon Vortex, equipado com barra pulverizadora de $14 \mathrm{~m}$, com acionamento hidráulico, e tanque com capacidade de $600 \mathrm{~L}$. As pontas de pulverização utilizadas foram AXI 110015 à pressão de 117,3 kPa, AXI 11002 e AXI 11003 a $276 \mathrm{kPa}$, as quais foram espaçadas de $0,50 \mathrm{~m}$ na barra, proporcionando a obtenção de 100; 200 e $300 \mathrm{~L} \mathrm{ha}^{-1}$ de calda, respectivamente. O diâmetro mediano volumétrico 
(DMV) das gotas produzidas pelas pontas de pulverização, nas respectivas pressões de trabalho, são $\geq 151$; 133, e entre 153 e 199 micrômetros, para as pontas 110015, 11002 e 11003, respectivamente, conforme especificações técnicas do fabricante. As pulverizações foram realizadas em 3-11-2005, tendo início às $9 \mathrm{~h}$ e término às $11 \mathrm{~h}$ da manhã, com o monitoramento das condições meteorológicas nesse período.

TABELA 1. Descrição dos tratamentos avaliados na deposição de um marcador, em plantas de arroz vermelho.

\begin{tabular}{ccc}
\hline Tratamentos & Tecnologia de pulverização & Volume $\left(\mathrm{L} \mathrm{ha}^{-1}\right)$ \\
\hline $\mathrm{T}_{1}$ & com assistência de ar & 100 \\
$\mathrm{~T}_{2}$ & & 200 \\
$\mathrm{~T}_{3}$ & sem assistência de ar & 300 \\
\hline $\mathrm{T}_{4}$ & (convencional) & 100 \\
$\mathrm{~T}_{5}$ & & 200 \\
$\mathrm{~T}_{6}$ & & 300 \\
\hline
\end{tabular}

A velocidade de deslocamento do conjunto trator-pulverizador foi de 1,33 $\mathrm{m} \mathrm{s}^{-1}$, determinada pelo tempo registrado para se deslocar $50 \mathrm{~m}$. Foram feitos o ajuste da pressão e a troca das pontas antecedendo as pulverizações de cada tratamento, separadamente, para cada ponta de pulverização envolvida neste estudo. A escolha da mesma velocidade de deslocamento do conjunto tratorpulverizador para diferentes pontas de pulverização foi necessária para minimizar a variabilidade na deposição da pulverização.

Para o mesmo volume aplicado, os tratamentos sem assistência de ar e com velocidade máxima (100\%) do ar na barra foram executados um após o outro, da seguinte forma: iniciou-se a pulverização com assistência de ar desligada, antes do início da parcela, adentrando-a já com a velocidade de deslocamento e vazão estabilizada; após o conjunto trator-pulverizador deslocar $15 \mathrm{~m}$ pulverizando, chegava-se ao término dessa parcela e, em área reservada para manobra entre parcelas $(5,0 \mathrm{~m})$ e com o equipamento em movimento, o operador acionava a assistência de ar, adentrando à próxima parcela com a assistência de ar ligada e estabilizada, interrompendo a pulverização ao término dessa parcela. As parcelas foram dimensionadas em $15 \mathrm{~m}$ de comprimento por igual distância de largura.

Para a avaliação quantitativa dos depósitos da pulverização, sob diferentes condições operacionais, utilizou-se como marcador o corante alimentício Azul Brilhante (PALLADINI et al., 2005) em solução aquosa $\left(1.500 \mathrm{mg} \mathrm{L}^{-1}\right)$. Em cada parcela, foram demarcadas, ao acaso, quatro subamostras com área equivalente a $1 \mathrm{~m}^{2}$ cada uma delas. De cada subamostra, foram colhidas seis plantas de arroz vermelho, totalizando 24 plantas por parcela.

As plantas colhidas foram colocadas, individualmente, em sacos plásticos devidamente identificados e acondicionados em caixa térmica (isopor). Esse material vegetal foi encaminhado ao Laboratório de Tecnologia de Aplicação de Defensivos Agrícolas para a remoção do marcador e posterior medição de área foliar de cada planta em um integrador de área foliar de bancada LICOR, modelo 3100.

Em cada saco plástico, foram colocados $10 \mathrm{~mL}$ de água destilada e procedeu-se a agitação por 30 segundos, visando à remoção do corante das plantas-alvo. A solução de lavagem foi colocada em vidros com capacidade de $20 \mathrm{~mL}$ e tampa de rosca, os quais foram mantidos à temperatura de $8 \pm 3{ }^{\circ} \mathrm{C}$ até o momento da análise.

As leituras (absorbância) dos depósitos do traçador foram realizadas em espectrofotômetro UV-VIS, Shimadzu, modelo CP 1601, equipado com filtro, no comprimento de onda de 630 nanômetros $(\mathrm{nm})$, conforme método desenvolvido por PALLADINI et al. (2005). 
Para determinar a concentração $\left(\mathrm{mg} \mathrm{L}^{-1}\right)$ dos depósitos, foi confeccionada a curva-padrão de linearidade com a mesma solução aplicada a campo. A curva-padrão de determinação da concentração do marcador na solução aplicada a campo foi determinada pelo modelo de regressão linear.

Considerando que a concentração de corante Azul Brilhante na calda pulverizada a campo foi de $1.500 \mathrm{mg} \mathrm{L}^{-1}$, para a transformação dos depósitos em microlitro $(\mu \mathrm{L})$, utilizou-se da seguinte equação:

em que,

$\mathrm{C}_{\mathrm{i}} \mathrm{V}_{\mathrm{i}}=\mathrm{C}_{\mathrm{f}} \mathrm{V}_{\mathrm{f}}$

$\mathrm{C}_{\mathrm{i}}$ - concentração inicial do traçador na calda, $\mathrm{mg} \mathrm{L}^{-1}$;

$\mathrm{V}_{\mathrm{i}}$ - volume retido pelo alvo, $\mu \mathrm{L}$;

$\mathrm{C}_{\mathrm{f}}$ - concentração detectada em densidade óptica, $\mathrm{mg} \mathrm{L}^{-1}, \mathrm{e}$

$\mathrm{V}_{\mathrm{f}}$ - volume de diluição da amostra de cada planta, $\mu \mathrm{L}$.

O efeito das tecnologias de aplicação (sem e com assistência de ar), combinadas aos volumes de 100; 200 e $300 \mathrm{~L} \mathrm{ha}^{-1}$ de calda sobre os depósitos da pulverização, foi avaliado estatisticamente, pelo teste $\mathrm{F}(\mathrm{p}<0,05)$. $\mathrm{O}$ desdobramento foi realizado somente quando a interação entre os fatores em estudo foi significativa a $5 \%$ de probabilidade.

A comparação dos dados médios dos depósitos do corante $\left(\mu \mathrm{L} \mathrm{cm}^{-2}\right)$ na superfície vegetal, para diferentes volumes e técnicas de aplicação, foi realizada pelo teste de Tukey, a 5\% de probabilidade.

\section{Experimento 2}

O efeito dos tratamentos utilizados no experimento 1 foi avaliado sobre o controle do arroz vermelho com o herbicida paraquat. Os mesmos procedimentos, equipamentos e tratamentos utilizados no experimento 1 foram adotados neste experimento com a adição das parcelas nãopulverizadas com o herbicida paraquat (testemunha). As pulverizações também foram realizadas na mesma data do experimento anterior (3-11-2005), após a remoção das plantas para a quantificação dos depósitos do marcador e secagem completa da pulverização da solução marcadora.

Para avaliar o efeito dessas tecnologias combinadas a diferentes volumes de aplicação no controle do arroz vermelho, utilizando-se do herbicida paraquat na dose de 3,0 $\mathrm{L} \mathrm{ha}^{-1}$, atribuíram-se notas, em percentagem de controle $(0$ a $100 \%)$ para as plantas infestantes, em cada parcela, decorrente do efeito dos respectivos tratamentos, comparado à parcela não-tratada (testemunha), conforme proposto pela Asociación Latinoamericana de Malezas - ALAM (1974). Para isso, quatro amostras com área equivalente a $1,0 \mathrm{~m}^{2}$ cada, selecionadas ao acaso, dentro de cada parcela, compuseram a percentagem média de controle dessas plantas.

As avaliações, em número de quatro, foram realizadas aos 3; 5; 7 e 9 dias após a aplicação (DAA).

A análise de variância do efeito das tecnologias combinadas a diferentes volumes no controle do arroz vermelho foi realizada pelo teste $\mathrm{F}$, e as médias, comparadas pelo teste de Tukey, a 5\% de probabilidade. O percentual médio de dessecação das plantas de arroz vermelho nos tratamentos, dentro de cada avaliação, foi comparado pelo teste de Tukey, a 5\% de probabilidade. A escolha dessa forma de análise, em relação àquela com a distribuição dos tratamentos no esquema fatorial, deveu-se ao fato de estar pulverizando herbicida com ação de contato, em que o fator tempo é menos importante, comparado ao uso de herbicida com ação sistêmica.

\section{RESULTADOS E DISCUSSÃO}

As condições meteorológicas mantiveram-se praticamente constantes durante a condução dos dois experimentos, com valores médios de temperatura, de umidade relativa do ar e de velocidade do vento, respectivamente, de $31,3{ }^{\circ} \mathrm{C}, 46,7 \%$ e $1,25 \mathrm{~m} \mathrm{~s}^{-1}$. O motivo de conduzir os experimentos 
próximos aos limites de temperatura e de umidade relativa do ar aceitáveis $\left(30{ }^{\circ} \mathrm{C}\right.$ e $55 \%$ UR) para realizar a pulverização deveu-se ao fato de dispor de tecnologia que possibilita a pulverização em condições adversas (STEVENSON \& JAMES, 1997 e JORGENSEN \& WITT, 2000), comparada à pulverização realizada por equipamentos convencionais (sem assistência de ar).

A concentração do depósito do marcador pulverizado a campo e retido pelas folhas de arroz vermelho foi determinada pela curva-padrão de linearidade, como segue: em que,

$$
\mathrm{y}=0,1099 \mathrm{x}+0,0009
$$

y - absorbância, e

$\mathrm{x}$ - concentração, $\mathrm{mg} \mathrm{L}^{-1}$.

O coeficiente de determinação $\left(\mathrm{R}^{2}\right)$ da curva foi 0,9994 para o intervalo da concentração do corante entre 0 e $5 \mathrm{mg} \mathrm{L}^{-1}$.

Os resultados da análise de variância para o depósito de corante por $\mathrm{cm}^{2}$ de área foliar do arroz vermelho, em função das técnicas de aplicação, volumes e para a interação técnica de aplicação x volumes, são apresentados na Tabela 2. Não se observou significância para técnicas de aplicação pelo teste $\mathrm{F}$, mostrando, assim, que não houve efeito desse fator sobre os depósitos da pulverização.

TABELA 2. Análise de variância do depósito de corante em função de diferentes técnicas e volumes de pulverização.

\begin{tabular}{cccc}
\hline FV & GL & F & Pr $>$ F \\
\hline Bloco & 3 & 3,070 & $0,0601 \mathrm{~ns}$ \\
Técnicas de pulverização & 1 & 0,024 & $0,8798 \mathrm{~ns}$ \\
Volumes & 2 & 11,628 & $0,000 *^{*}$ \\
TP x V & 2 & 6,250 & $0,0106^{*}$ \\
Resíduo & 15 & & \\
\hline C.V. $(\%)$ & 26 & & \\
\hline
\end{tabular}

ns - não-significativo $(\mathrm{p}<0,05) ; *$ Significativo a $5 \%$ de probabilidade.

Para volumes e a interação entre técnicas de aplicação (com e sem ar) x volumes, houve significância a $5 \%$ de probabilidade (Tabela 2).

As médias dos depósitos das pulverizações realizadas com e sem assistência de ar junto à barra não diferiram significativamente, a 5\% de probabilidade. Já para os volumes de pulverização, verificou-se diferença significativa entre os valores médios dos depósitos dos maiores volumes (200 e $\left.300 \mathrm{~L} \mathrm{ha}^{-1}\right)$ em relação ao menor volume (100 L ha ${ }^{-1}$ ) (Tabela 3).

TABELA 3. Médias dos depósitos $\left(\mu \mathrm{L} \mathrm{cm}^{-2}\right)$ obtidos com diferentes técnicas e volumes de pulverização.

\begin{tabular}{cc}
\hline Técnicas de pulverização & Médias dos Depósitos $\left(\mu \mathrm{L} \mathrm{cm}^{-2}\right)^{1}$ \\
Com ar & $0,475 \mathrm{a}$ \\
Sem ar & $0,467 \mathrm{a}$ \\
\hline DMS & 0,107 \\
\hline Volume & Médias dos Depósitos $\left(\mu \mathrm{L} \mathrm{cm}^{-2}\right)$ \\
100 & $0,303 \mathrm{a}$ \\
200 & $0,521 \mathrm{~b}$ \\
300 & $0,588 \mathrm{~b}$ \\
\hline DMS & 0,160 \\
\hline
\end{tabular}

\footnotetext{
${ }^{1}$ Médias seguidas pelas mesmas letras não diferem entre si, pelo teste de Tukey, a 5\% de significância.
} 
Os valores médios dos depósitos da calda $\left(\mu \mathrm{L} \mathrm{cm} \mathrm{cm}^{-2}\right)$ para a interação volumes $(100 ; 200$ e $300 \mathrm{~L} \mathrm{ha}^{-1}$ ) e técnicas de pulverização (com e sem assistência de ar) são apresentados na Tabela 4.

Ao comparar as técnicas de pulverização dentro de cada volume de calda (letras maiúsculas), pode-se verificar que não houve diferença significativa somente para o volume de $200 \mathrm{~L} \mathrm{ha}^{-1}$. Para o volume de $100 \mathrm{~L} \mathrm{ha}^{-1}$, na ausência da assistência de ar, constatou-se maior depósito do traçador em plantas de arroz vermelho sob cultivo de nabo forrageiro (Tabela 4). No volume de $300 \mathrm{~L} \mathrm{ha}^{-1}$, ocorreu o inverso do tratamento anterior, em que a assistência de ar proporcionou incremento dos depósitos em relação à pulverização convencional (sem ar). Via de regra, os maiores volumes proporcionam maiores depósitos na mesma velocidade de aplicação (MATTHEWS \& HISLOP, 1993; MATTHEWS, 2000). Por outro lado, os níveis dos depósitos nas plantas-alvo não dependem apenas do equipamento aplicador, mas também da idade, do tipo da cultura, densidade, condição operacional e do tipo de ponta de pulverização (COOKE et al.,1990).

Quando se comparam os volumes de calda com o uso da assistência de ar (Tabela 4), constatam-se depósitos do corante significativamente maiores para os volumes de 200 e $300 \mathrm{~L} \mathrm{ha}^{-1}$ em relação ao volume aplicado de $100 \mathrm{~L} \mathrm{ha}^{-1}$.

As médias das percentagens de controle das plantas de arroz vermelho são apresentadas na Tabela 5. Na primeira avaliação de controle de Oryza sativa L., o volume de $300 \mathrm{~L} \mathrm{ha}^{-1}$ sobressaiuse tanto na presença de assistência de ar quanto na ausência dessa tecnologia, não diferindo entre si; já os tratamentos com 100 e $200 \mathrm{~L} \mathrm{ha}^{-1}$ obtiveram as piores percentagens de controle.

$\mathrm{Na}$ segunda e terceira avaliações, respectivamente, aos 5 e 7 DAA, os tratamentos de $200 \mathrm{~L} \mathrm{ha}^{-1}$ com assistência de ar e $300 \mathrm{~L} \mathrm{ha}^{-1} \mathrm{com} \mathrm{e} \mathrm{sem} \mathrm{assistência} \mathrm{de} \mathrm{ar} \mathrm{não} \mathrm{diferiram}$ significativamente entre si, porém diferiram da pulverização sem assistência de ar com $100 \mathrm{~L} \mathrm{ha}^{-1}$. $\mathrm{Na}$ quarta e última avaliação, aos 9 DAA, a percentagem de controle, aplicando $100 \mathrm{~L} \mathrm{ha}^{-1} \mathrm{com}$ assistência de ar, não diferiu daquelas obtidas com os volumes de 200 e $300 \mathrm{~L} \mathrm{ha}^{-1}$ utilizando essa tecnologia.

TABELA 4. Efeito de técnicas de pulverização (com e sem ar) e volumes de calda (100; 200 e $\left.300 \mathrm{~L} \mathrm{ha}^{-1}\right)$ sobre os depósitos do corante $\left(\mu \mathrm{L} \mathrm{cm}^{-2}\right)$, em plantas de arroz vermelho.

\begin{tabular}{ccc}
\hline \multirow{2}{*}{ Volume $\left(\mathrm{L} \mathrm{ha}^{-1}\right)$} & \multicolumn{2}{c}{ Técnicas de Aplicação } \\
\cline { 2 - 3 } & Com ar & Sem ar \\
\hline 100 & $0,187 \mathrm{Bb}$ & $0,418 \mathrm{Aa}$ \\
200 & $0,550 \mathrm{Aa}$ & $0,492 \mathrm{Aa}$ \\
300 & $0,686 \mathrm{Aa}$ & $0,489 \mathrm{Ba}$ \\
\hline
\end{tabular}

Médias seguidas das mesmas letras maiúsculas na linha e minúsculas na coluna não diferem entre si, pelo teste de Tukey, a $5 \%$ de probabilidade.

Apesar de se obterem depósitos similares do corante, sem a assistência de ar, para os diferentes volumes (Tabela 4), maiores percentagens de controle do arroz vermelho foram obtidas com maiores volumes de calda (Tabela 5). Provavelmente, a eficiência de captura das gotas de menores diâmetros por alvos predominantemente verticais (plantas de arroz vermelho com 2 a 4 folhas), na pulverização do menor volume de calda, contribuiu para obtenção de níveis similares de depósitos do corante aos obtidos com 200 e $300 \mathrm{~L} \mathrm{ha}^{-1}$, sem a assistência de ar junto à barra pulverizadora, mesmo em condições meteorológicas críticas (temperatura maior que $30^{\circ} \mathrm{C}$ e UR do ar menor que 55\%) em época de veranico.

A rigor, apesar de pequenas variações no DMV das gotas produzidas pelas diferentes pontas, elas são classificadas como gotas finas (MATTHEWS, 2000). No entanto, o uso da assistência de ar influenciou negativamente nos níveis dos depósitos para o menor volume pulverizado. A menor cobertura vegetal com a cultura do nabo forrageiro, nesse estádio de desenvolvimento, bem como a possibilidade de ocorrência da deflexão do ar pela superfície do solo podem ter contribuído para a retenção de parte dessas gotas nessa espécie vegetal e, dessa forma, reduzindo os depósitos sobre as plantas de arroz vermelho. 
Quando o solo está coberto pela vegetação, a assistência de ar auxilia aquelas gotas de menor momento (menor quantidade de movimento) a penetrar no dossel da cultura (MATTHEWS, 2000; RAETANO \& BAUER, 2003), mas, nesse caso em que a cultura do nabo forrageiro estava com menor densidade foliar, a assistência de ar, por deflexão, pode ter contribuído para que parte dessas gotas finas fosse captada pelas plantas do nabo forrageiro, reduzindo os depósitos do corante nas plantas de arroz vermelho, especialmente quando o menor volume foi pulverizado. Porém, a quantidade do herbicida que atingiu essas plantas infestantes, foi suficiente para proporcionar níveis de controle semelhantes aos obtidos com os maiores volumes de pulverização.

Apesar de a assistência de ar influenciar positivamente nos depósitos das gotas oriundas dos volumes pulverizados com as pontas AXI 11002 e 11003 , respectivamente, 200 e $300 \mathrm{~L} \mathrm{ha}^{-1}$, a maior percentagem de controle com o maior volume pulverizado, sem assistência de ar, parece estar relacionado ao maior volume e melhor distribuição desse volume em relação aos demais tratamentos.

Desempenho comparável na dessecação de aveia-preta (Avena strigosa Schreb) foi obtido por GARCIA et al. (2004), utilizando a mistura dos herbicidas dicloreto de paraquat (400 $\left.\mathrm{g} \mathrm{ha}^{-1}\right) \mathrm{e}$ diuron $\left(200 \mathrm{~g} \mathrm{ha}^{-1}\right)$, no volume de $100 \mathrm{~L} \mathrm{ha}^{-1}$, em presença da assistência de ar junto à barra pulverizadora, comparado ao dobro desse volume na ausência dessa tecnologia.

TABELA 5. Percentagem média de dessecação de arroz vermelho em função da aplicação com e sem assistência de ar e diferentes volumes de calda. $(n=4)$.

\begin{tabular}{ccccc}
\hline \multirow{2}{*}{$\begin{array}{c}\text { Volume } \\
\left(\mathrm{L} \mathrm{ha}^{-1}\right)\end{array}$} & \multicolumn{4}{c}{ Avaliação da Dessecação (\%) } \\
\cline { 2 - 5 } 100 & 3 DAA & DAA & 7 DAA & 9 DAA \\
\cline { 2 - 5 } 200 & $30,00 \mathrm{c}$ & $41,25 \mathrm{~d}$ & $51,25 \mathrm{~d}$ & $73,75 \mathrm{c}$ \\
300 & $25,00 \mathrm{c}$ & $45,00 \mathrm{~cd}$ & $60,00 \mathrm{~cd}$ & $77,50 \mathrm{bc}$ \\
& $43,75 \mathrm{ab}$ & $56,25 \mathrm{ab}$ & $71,25 \mathrm{ab}$ & $85,00 \mathrm{a}$ \\
\cline { 2 - 5 } 100 & $33,75 \mathrm{bc}$ & $50,00 \mathrm{bc}$ & $63,75 \mathrm{bc}$ & $82,50 \mathrm{ab}$ \\
200 & $35,00 \mathrm{bc}$ & $52,50 \mathrm{abc}$ & $70,00 \mathrm{ab}$ & $85,00 \mathrm{a}$ \\
300 & $46,25 \mathrm{a}$ & $60,00 \mathrm{a}$ & $76,25 \mathrm{a}$ & $88,75 \mathrm{a}$ \\
\hline C.V. $(\%)$ & 13,13 & 6,47 & 6,08 & 3,40 \\
DMS & 10,74 & 7,56 & 9,14 & 6,40 \\
\hline
\end{tabular}

Médias seguidas de mesma letra na coluna não diferem entre si, pelo teste de Tukey, a 5\% de probabilidade.

\section{CONCLUSÕES}

A assistência de ar junto à barra de pulverização incrementou os depósitos do corante marcador Azul Brilhante em plantas de arroz vermelho sob o cultivo do nabo forrageiro, em área de várzea, para o volume de $300 \mathrm{~L} \mathrm{ha}^{1}$.

Níveis satisfatórios de controle do arroz vermelho sob a cultura do nabo forrageiro foram obtidos com o herbicida paraquat, na dose de 3,0 $\mathrm{L} \mathrm{ha}^{-1}$.

A assistência de ar permitiu reduzir o volume de pulverização de 300 para $100 \mathrm{~L} \mathrm{ha}^{-1}$, com boa eficiência de controle do arroz vermelho.

\section{REFERÊNCIAS}

ALAM. ASOCIACIÓN LATINOAMERICANA DE MALEZAS. Recomendaciones sobre unificación de evaluación en ensayos de control de malezas. ALAM, Bogotá, v.1, n.1, p.35-8, 1974.

BAUER, F.C.; RAETANO, C.G. Assistência de ar e perdas na deposição de produtos fitossanitários em pulverizações na cultura da soja. Scientia Agricola, Piracicaba, v.57, n.2, p. 271 6, 2000. 
CONSTANTIN, J.; OLIVEIRA JÚNIOR, R.S.; FAGLIARI, J.R.; PAGLIARI, P.H.; ARANTES, J.G.Z.; CAVALIERI, S.D.; FRAMESQUI, V.P.; GONÇALVES, D.A. Efeito de subdoses de 2,4-D na produtividade do algodão e suscetibilidade da cultura em função de seu estádio de desenvolvimento. Engenharia Agrícola, Jaboticabal, v.27, n.esp., p.24-9, 2007.

COOKE, B.K.; HISLOP, E.C.; HERRINGTON, P.J.; WESTERN, N.M.; HUMPHERSONJONES, F. Air assisted spraying of arable crops, in relation to deposition, drift and pesticide performance. Crop Protection, Oxford, v.9, n.4, p. 303-11, 1990.

FURNESS, G.O. A comparison of simple bluff plate and axial fans for air-assisted, high- speed, low - volume spray application to wheat and sunflower plants. Journal of Agricultural Engineering Research, London, v.48, n.1, p. 57-75, 1991.

GARCIA, L.C.; RAETANO, C.G.; JUSTINO, A.; PURÍSSIMO, C. Dessecação da aveia-preta (Avena strigosa Schreb) com herbicida de contato, em presença ou não de assistência de ar junto à barra do pulverizador, em diferentes volumes de calda. Engenharia Agrícola, Jaboticabal, v.24, n.3, p.758-63, 2004.

HISLOP, E.C. Air assisted crop spraying: an introductory review. In: LAVERS, A.; HERINGTON, P.; SOUTHCOMBE, E. S. E. Air-assisted spraying in crop protection. Swansea: British Crop Protection Council, 1991. p.3-14 (BCPC Monograph, 46).

JORGENSEN, L.; WITT, K.L. Spraying and the impact on the environment: spraying technique in relation to approval and use of pesticides in Northern Europe. In: HARDI INTERNATIONAL APPLICATION TECHNOLOGY COURSE, 2000, Taastrup. v.1, chap.2, p.4-16.

MATTHEWS, G.A. Pesticide application methods. $3^{\text {rd }}$ ed. London: Balckwell, 2000. 432 p.

MATTHEWS, G.A.; HISLOP, E.C. Application technology for crop protection. Wallingford: CAB INTERNATIONAL, 1993. 359 p.

OLIVEIRA JÚNIOR, R.S.; CONSTANTIN, J.; BRANDÃO FILHO, J.U.T.; CALLEGARI, O.; PAGLIARI, P.H.; CAVALIERI, S.D.; FRAMESQUI, V.P.; CARREIRA, S.A.M.; ROSO, A.C. Efeito de subdoses de 2,4-D na produtividade de uva Itália e suscetibilidade da cultura em função de seu estádio de desenvolvimento. Engenharia Agrícola, Jaboticabal, v.27, n.esp., p.35-40, 2007.

PALLADINI, L.A.; RAETANO, C.G.; VELINI, E.D. Choice of tracers for the evaluation of spray deposits. Scientia Agricola, Piracicaba, v.62, n.5, p. 440-5, 2005.

RAETANO, C.G.; BAUER, F.C. Efeito da velocidade do ar em barra de pulverização na deposição de produtos fitossanitários em feijoeiro. Bragantia, Campinas, v.62, n.2, p.329-34, 2003.

STEVENSON, W.R.; JAMES, R.V. Evaluation of different sprayers technologies for fungicide application to control early and late blights. Vegetable Diseases Control Trials, Wisconsin, p.21-8, 1997. Disponível em: http://www.plantpath.wisc.edu/wivegdis. Acesso em: 15 jun. 2005. 